\title{
Nanometer-scale Analysis of Space-weathered Lunar Regolith by Atom Probe Tomograhpy
}

Josiah Lewis $^{1}$, Dieter Isheim ${ }^{2}$, Bradley Jolliff ${ }^{1}$, David Seidman ${ }^{2}$, Ryan Ogliore ${ }^{1}$ and Jeffrey Gillis-Davis ${ }^{1}$

${ }^{1}$ Washington University-St. Louis, St. Louis, Missouri, United States, ${ }^{2}$ Northwestern University, Evanston, Illinois, United States

Solar wind implantation and micrometeorite impacts (i.e., space weathering) alter the surfaces of airless bodies throughout the solar system [1]. In lunar regolith, space weathering produces complex microstructures, specifically amorphous rims that coat grains and often contain nanophase and microphase metallic $\mathrm{Fe}$ (npFe and $\mathrm{mpFe}$ ) [2]. FeS inclusions were found on the surface of dust particles returned from asteroid Itokawa [3]. In addition to informing models of space weathering, detailed knowledge of the microstructure, particle size distributions, and number densities of $n p F e$ and $m p F e$ is necessary for interpreting and modeling near-infrared reflectance spectra obtained by remote sensing of the lunar surface and other bodies in the solar system [4].

We use atom-probe tomography (APT) [5], with 3D nano-scale spatial resolution and single atom sensitivity, to characterize the composition of the surface layer of a lunar regolith agglutinate from sample 10084, returned by the Apollo 11 mission in 1969. SEM imaging and FIB lift-out techniques enable targeted APT analysis and contextual interpretation of the results [6].

APT analyses of two sample volumes from the surface of the same agglutinate particle reveal distinct compositional layering and a variety of heterogeneous inclusions with nanometer-scale characteristic dimensions. One APT analysis reveals two $20 \mathrm{~nm}$ diameter npFe grains near the surface, label ' $\mathrm{A}$ ' in Fig. 1. These npFe grains contain a comparatively high Ni concentration (5-6 at.\%), similar to kamacite, and thus provide evidence for meteoritic origin. Approximately fifty smaller npFe particles with $\sim 3-5 \mathrm{~nm}$ diameter and with a lower Ni concentration are located in a layer at 20-60 nm depth, label 'B' in Fig. 1. Aluminum, $\mathrm{Ca}$, and $\mathrm{Mg}$ are depleted near the surface and the relative $\mathrm{H}$ concentration is higher in the same region, consistent with a solar-wind altered rim.

A second APT analysis was targeted on a $\sim 0.5 \mu \mathrm{m}$ diameter mpFe particle that was easily identified by back-scatter contrast in SEM as a region with higher than average atomic number $(Z)$, at a location $\sim 5$ $\mu \mathrm{m}$ away from the first APT analysis of this same agglutinate grain. This mpFe particle has a lower Ni content $(\mathrm{Ni} / \mathrm{Fe}<0.01)$ but regions enriched in $\mathrm{C}(\mathrm{C} / \mathrm{Fe} \sim 0.12)$, label ' $\mathrm{A}$ ' in Fig. 2, enriched in $\mathrm{P}(\mathrm{P} / \mathrm{Fe} \sim$ 0.03), label ' $\mathrm{B}$ ' in Fig. 2, and a 40 nm long ellipsoidal FeS-type inclusion, label ' $\mathrm{C}$ ' in Fig. 2. The $\mathrm{C}, \mathrm{P}$, and $\mathrm{S}$-rich inclusions may also indicate meteoritic contamination.

The evidence for a meteoritic component to $\mathrm{npFe}$ and $\mathrm{mpFe}$ is an unexpected finding that runs counter to most models describing their formation [7]. 


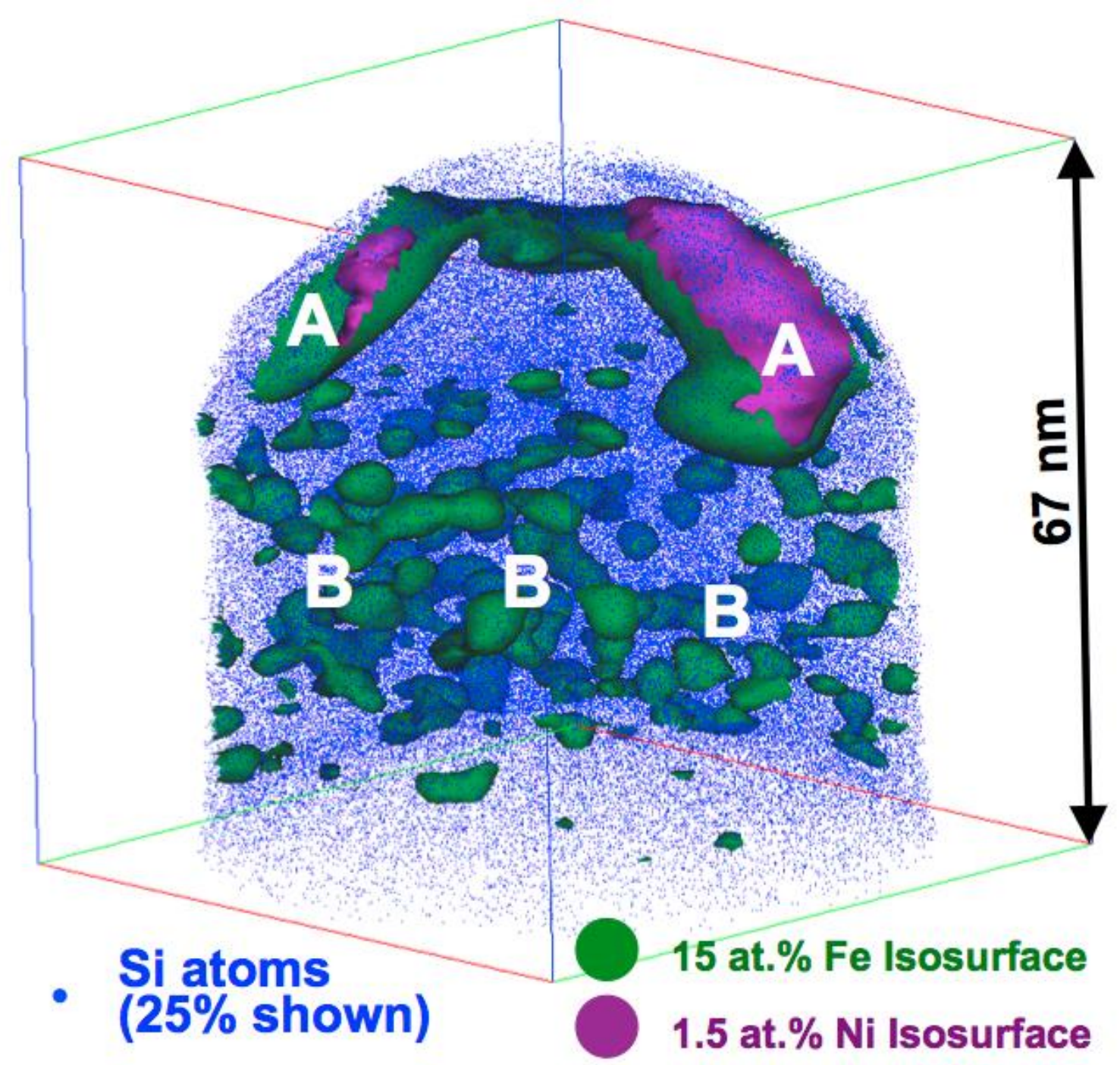

Figure 1. 3D atom-by-atom APT reconstruction of a volume from the surface layer of a lunar agglutinate particle. Two nanophase Fe particles (label 'A'), delineated by a 15 at.\% Fe isoconcentration surface, are present in a layer $\sim 7-22 \mathrm{~nm}$ from the surface (top). These larger npFe particles contain up to $\sim 5-6$ at.\% $\mathrm{Ni}$, shown by a 1.5 at.\% $\mathrm{Ni}$ isoconcentration surface. Approximately 50 smaller npFe 3-5 nm in diameter (label 'B') are located in a layer at 20-60 $\mathrm{nm}$ depth from the surface. 


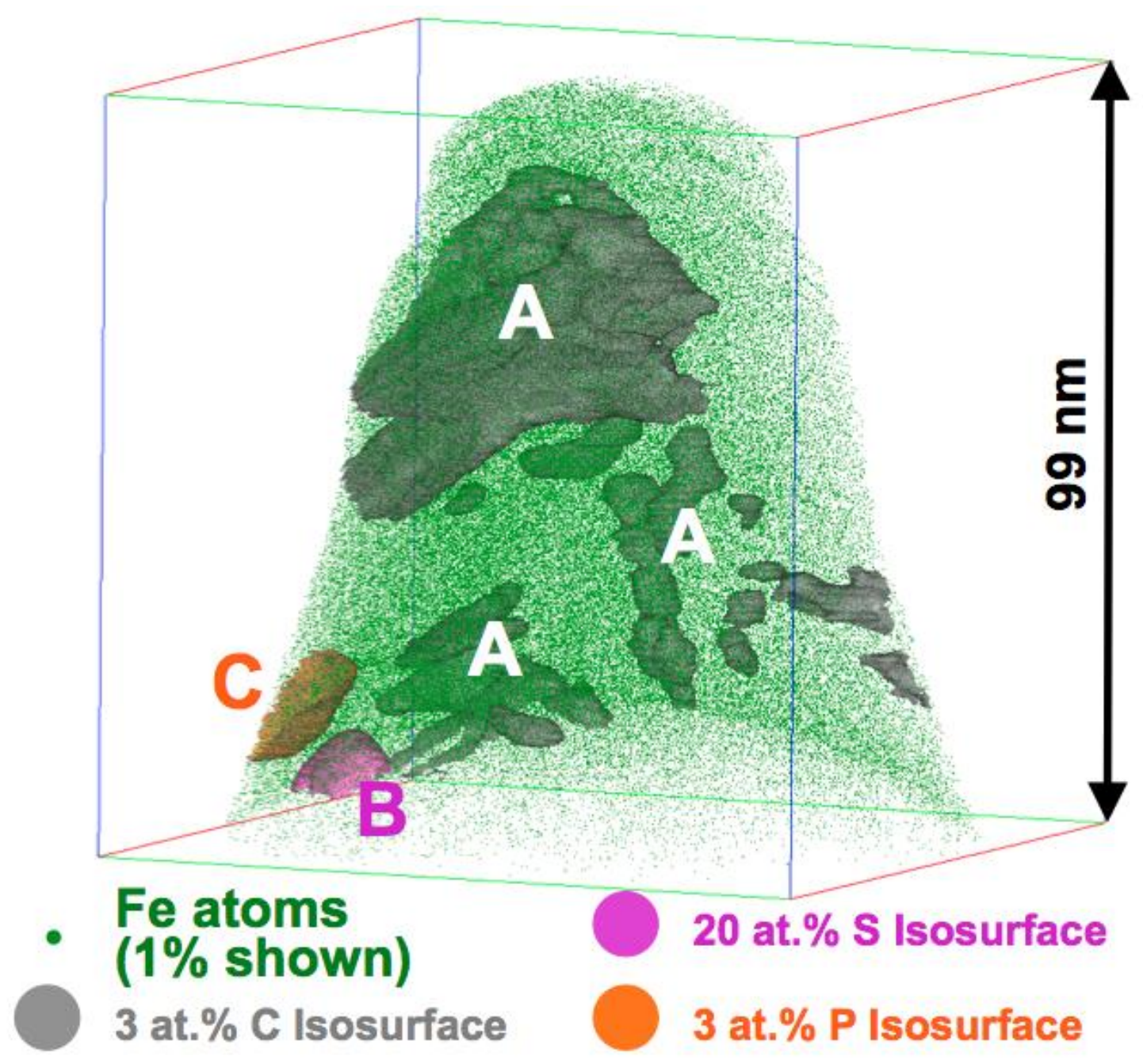

Figure 2. APT reconstruction of a volume in a microphase Fe particle at the surface of the same lunar agglutinate particle but about $5 \mu \mathrm{m}$ away from the volume displayed in Fig. 1. This mpFe particle contains regions rich in carbon (label ' $\mathrm{A}$ '), delineated by a 3 at. $\% \mathrm{C}$ isoconcentration surface. Additionally, a region enriched in $\mathrm{P}$ (label 'B', 3 at.\% $\mathrm{P}$ isoconcentration surface), and a region rich in $\mathrm{S}$ (label 'C', 20 at.\% S isoconcentration surface), possibly a FeS-type inclusion, are detected in this mpFe grain.

References

[1] J Bibring et al., Science 175 (1972), pp. 753-755.

[2] LP Keller, DS McKay, Science 261 (1993), pp. 1305-1307.

[3] T Noguchi et al., Meteoritics \& Planetary Scienc. 49 (2014), pp. 188-214.

[4] B Hapke, Journal of Geophysical Research: Planets 106(E5) (2001), pp. 10039-10073.

[5] DJ Larson et al., Local Electrode Atom Probe Tomography: A User's Guide, Springer (2013).

[6] JB Lewis et al., Apollo 11 Agglutinate Investigated by Atom-probe Tomography. EPSC-DPS Joint Meeting 2019. 13, EPSC-DPS2019-75-2.

[7] Techniques used in this work were developed using support by NASA (grant number NNX16AD26G). APT was performed with the LEAP5000 XS at Northwestern University (NUCAPT), acquired with grants from NSF-MRI (DMR-0420532) and ONR-DURIP (N00014-0400798, N00014-0610539, N000140910781, N00014-1712870). NUCAPT received support from the MRSEC program (NSF DMR- 
1720139) at the Materials Research Center, the SHyNE Resource (NSF ECCS-1542205), and the Initiative for Sustainability and Energy (ISEN) at Northwestern University. 